\title{
Managing Subacute Subperiosteal Hematoma with High Intraocular Pressure: A Case Report
}

\author{
Hashim SE, ${ }^{a}$ Fatisha A, ${ }^{\mathrm{b}}$ Nazri $M N^{\mathrm{c}}$ \\ ${ }^{a}$ Ophthalmology Department, Medical Faculty, Universiti Sultan Zainal Abidin, Jalan Sultan Mahmud, \\ 20400, Kuala Terengganu, Terengganu, Malaysia. \\ ${ }^{b}$ Ophthalmology Department, Hospital Sultanah Nur Zahirah, Jalan Sultan Mahmud, 20400, Kuala \\ Terengganu, Terengganu, Malaysia. \\ 'Jabatan Pengimejan Diagnostik, Hospital Sultanah Nur Zahirah, Jalan Sultan Mahmud, 20400, Kuala \\ Terengganu, Terengganu, Malaysia.
}

\section{ABSTRACT}

Subperiosteal haematoma of the orbit is an uncommon complication of maxillofacial trauma, hence easily missed. It usually presents subacutely with proptosis and diplopia. In our case, the subperiosteal haematoma is complicated with high intraocular pressure, necessisating measures to reduce the intraocular pressure. Unresponsive to only medical treatment, surgical evacuation was carried out in this patient. Removal of the clot finally relieved the intraocular pressure and simultaneously improved the proptosis and the cumbersome diplopia.

Keywords: Subperiosteal hematoma of the orbit, Subacute Proptosis post trauma, High intraocular pressure in subperisoteal hematoma, Surgical evacuation subperiosteal hematoma, Extradural hematoma and subperiosteal hematoma

\section{INTRODUCTION}

Causes of proptosis after maxillofacial trauma include orbital fracture, retro-bulbar haemorrhage, carotico-cavernous fistula, orbital emphysema, orbital abscess and subperiosteal haematoma of the orbit. The latter is rare complication of maxillofacial trauma, but when present, commonly presents with proptosis. ${ }^{1}$ Sub periosteal haematoma of the orbit had also been reported from nontraumatic causes, such as suicide, sinusitis and scuba diving, ${ }^{2-3}$ underlying blood clotting disorder and idiopathic causes. Apart from proptosis, subperiosteal haematoma is also associated with diplopia, malposition of the eye, ophthalmoplegia and periorbital haematoma. ${ }^{4}$ Our extensive literature search thus far has not led to any previously reported case of subperiosteal haematoma complicated by high intraocular pressure. In this report, we present a patient who developed subacute sub periosteal haematoma after

Corresponding author:

Dr. Syaratul Emma Binti Hashim

Medical Lecturer \& Consultant Ophthalmologist

Ophthalmology Department, Medical Faculty,

Universiti Sultan Zainal Abidin

Jalan Sultan Mahmud, 20400, Kuala Terengganu,

Terengganu, Malaysia

Tel no : $+609-6275575$

Fax no : +60909-6275745

Mobile no: 019-9852700

Email : emmahashim@unisza.edu.my/

dremma73@gmail.com a trivial injury. Complicated with persistent high intraocular pressure and unresolved proptosis, surgical evacuation was carried out. The patient improved tremendously after the procedure and intraocular pressure was normalised.

\section{CASE REPORT}

An 11- year-old boy had a history of fall from his bicycle and landed with his face onto the ground. He complained of mild frontal headache, but no loss of consciousness, no bleeding from oral, nasal or auricular orifices, nor was there any nausea or vomiting. He has no diplopia or blurring of vision at that time. Physical examination revealed right periorbital swelling and mild facial bruises and abrasions. The eye was not proptosed and both eyes were orthophoric. The rest of the anterior and posterior segment eye examinations were unremarkable. Orbital radiograph was normal with no bony discontinuity. He was then treated conservatively as an outpatient. However, on day 5 post trauma, the patient presented again for a sudden onset of proptosis in the right eye associated with diplopia. He also complained of mild throbbing eye pain. His vision was unaffected and there were no other neurological symptoms. He also denied any previous history of easy bruising or spontaneous bleeding.

On examination, patient was fully conscious, with a normal Glasgow coma scale. His visual acuity was $6 / 6$ in both eyes. Both pupils were equal and reactive to light. Relative afferent pupillary defect 
was negative. There was marked non-axial proptosis of the right eye, which was displaced inferiorly (Figure 3a). By Hertel exophthalmometer, the right eye measured at $8 \mathrm{~mm}$ difference compared to the left eye, indicating significant proptosis. No pulsation or bruit was detected in the proptosed right eye. There was complete restriction of elevation and marked limitation of depression, adduction and abduction of the right eye. Hess charting also revealed similar marked restriction of all gazes in the right eye. Vertical diplopia was reported in all fields of gazes. Anterior segment examination revealed white conjunctiva and clear cornea. The anterior chamber of both eyes was deep, with no cells or pigments seen. The intraocular pressure was however raised in the right eye which measured at $35 \mathrm{mmHg}$, compared to
$12 \mathrm{mmHg}$ in the left eye. Funduscopy revealed hyperaemic disc with cup-disc ratio of 0.1 . There was no venous engorgement, and the macula was not oedematous. The rest of the fundus was normal. Other examinations were unremarkable with no neurological deficits.

Computed tomography (CT) demonstrated a postseptal haematoma along the right orbital roof measuring $30.0 \mathrm{~mm}(\mathrm{TX}) \times 32.0 \mathrm{~mm}$ (AP) $\times 17.0 \mathrm{~mm}$ (CC) with inferior displacement of the globe and stretching the optic nerve (Figure 1a and Figure 2a). A small subfrontal extradural haematoma was also seen. There was no fracture seen. Blood investigations were performed to rule out any bleeding or coagulation disorder but no abnormality was reported.

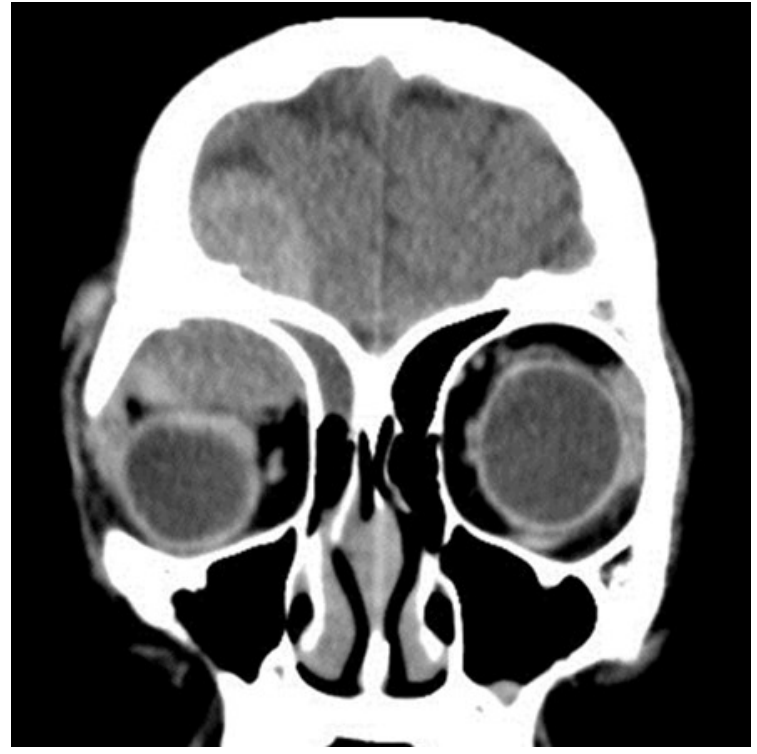

Figure 1a

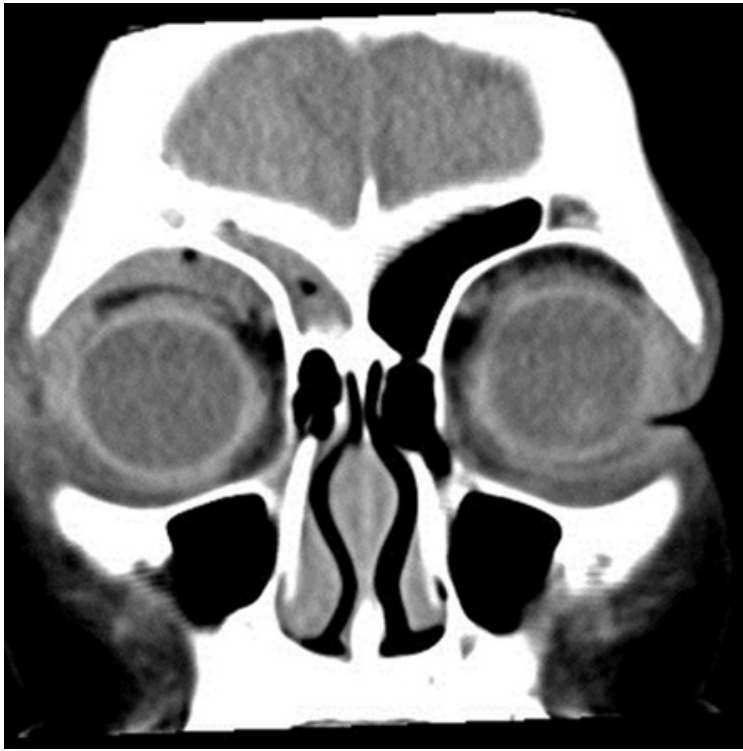

Figure $1 \mathrm{~b}$

Figure 1a: Coronal view of pre-operative CT scan showing the superior subperiosteal hematoma pushing the globe inferiorly Figure $1 \mathrm{~b}$ : Postoperative $\mathrm{CT}$
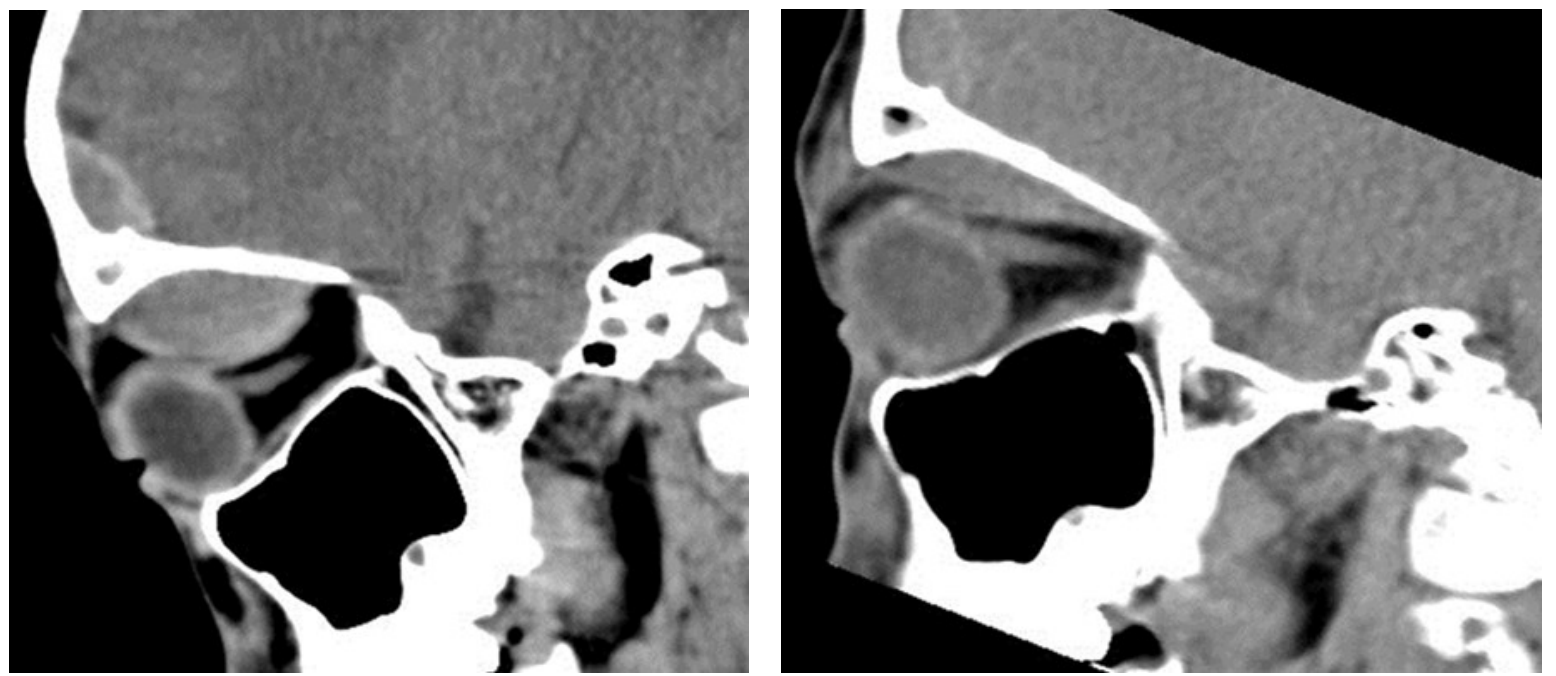

Figure 2a: Sagittal view of pre-operative $\mathrm{CT}$ with hematoma compressing and stretching the optic nerve. Figue $2 \mathrm{~b}$ : The release of optic nerve compression postoperatively 
Patient was then admitted and medication was prescribed to reduce the intraocular pressure. He was started on oral Acetazolamide $125 \mathrm{mg}$ qid, Gutt Timolol $0.5 \%$ bd and Gutt Brimonidine $0.2 \%$ for the right eye. Daily monitoring of visual acuity, relative afferent pupillary defect and intraocular pressure was performed. He was also started on oral Prednisolone $1 \mathrm{mg} / \mathrm{kg} /$ day. Visual monitoring was normal and the relative afferent pupillary defect remained negative. After 3 days on oral and topical medication, the intraocular pressure remained at more than $30 \mathrm{mmg} \mathrm{Hg}$, and there was no sign of improvement in the degree of proptosis and diplopia.

With these clinical indications, surgical evacuation was carried out after 8 days of trauma. Under general anaesthesia, drainage was performed through an incision line made above the lateral border of the right eyebrow. After a second incision made on the periosteum, posterior to superior orbital rim, there was spontaneous drainage of hemolysed clot through the wound. A capsulated haemolysed blood clot was also removed and the estimated blood clot drained was $10 \mathrm{ml}$. Haemostasis was secured and the wound was closed with Ethilon 6-0.

On the first postoperative day, the proptosis markedly reduced from $8 \mathrm{~mm}$ to $2 \mathrm{~mm}$ (Figure $3 \mathrm{~b}$ ). There was no more diplopia and movement of the right eye was almost full in all directions. All intraocular lowering agents were withdrawn and the pressure normalized to less than $15 \mathrm{mmHg}$. A repeat CT scan done 2 days postoperatively, showed some residual haematoma, measuring $17.8 \mathrm{~mm}$ (TX) $\mathrm{x}$ $25.9 \mathrm{~mm}(\mathrm{AP}) \times 5.6 \mathrm{~mm}$ (CC) with a few air pockets seen within the haematoma likely from the surgical intervention (Figure $1 \mathrm{~b}$ and Figure $2 \mathrm{~b}$ ).

He was reviewed in the clinic after 1 month with no ocular complains. Vision was $6 / 6$ in both eyes and the intraocular pressure was normal. Patient was not on any medication. Visual field examination revealed no abnormality and the extraocular movement was full. Both eyeballs were also in the same plane. Gonioscopy was performed and there was no sign of angle recession in his right eye.

\section{DISCUSSION}

Uncommonly mentioned in the textbook, subperiosteal haematoma is one complication that should not be overlooked when dealing with maxillofacial trauma. Its commoner counterpart is retrobulbar haemorrhage, which can occur after a maxillofacial trauma or surgery to the orbit or paranasal sinuses. The rapid progression and dramatic clinical features of retrobulbar haemorrhage requires prompt action to be taken to save the eye sight. ${ }^{5}$ The creation of compartment syndrome secondary to accumulation of blood within postseptal space may damage the optic nerve and the blood supply to the eye.
In subperiosteal haematoma, the acute loss of vision due to optic nerve head ischemia is a less worrying complication. The clinical course is less rapid compared to retro-bulbar haemorrhage as the haematoma may take a few days to develop. It usually presents with proptosis, vertical globe displacement and diplopia. ${ }^{1}$ Subperiosteal haematoma can be well demonstrated on CT scan as a sharply defined hyperdense, extraconal nonenhancing mass with broad base towards the bone and displacing periorbita centrally. ${ }^{6}$ Fractured wall of the orbit, cavernous sinus fistula and emphysema of the orbit are the other differential diagnoses that should be considered in subacute proptosis post trauma.

Here, we highlighted a case of subacute proptosis secondary to trauma with a persistent increase in the intraocular pressure. High intraocular pressure secondary to proptosis has been well documented in the literature ${ }^{7}$ and can be explained by 2 main factors. The first factor is the effect of proptosis on the physiological conventional aqueous drainage. Outflow of aqueous via this conventional route is passive and largely depends on the difference between the intraocular pressure and episcleral venous pressure. Proptosis secondary to blood clot, exudates, inflammatory cells, mass or muscle hypertrophy within the orbit can cause obstruction to the episcleral venous outflow. This will lead to an inevitable increase in episcleral venous pressure resulting in decrease aqueous drainage and hence increased intraocular pressure.

The second factor is the anatomically confined space of the orbital cavity. Bounded at the sides by seven rigid bones and anteriorly by the orbital septum, this space can only accommodate a small increase in its content volume. As the volume accumulates further, the high intraorbital pressure will impede the venous drainage, with consequent elevation in episcleral venous pressure and eventual increase in the intraocular pressure.

A persistently high intraocular pressure may lead to progressive optic neuropathy and ultimately irreversible blindness if poorly managed. Lowering the intraocular pressure by medical treatment seems an initial appropriate management, but removing the primary blockage of venous drainage is the ultimate management to improve the episcleral venous flow. The recorded pressure in our case remained high and did not respond much with pressure lowering agents. Nevertheless, the pressure was not alarmingly high and further "wait and see" period could be extended. However, due to the need to allow the patient to return to school immediately as well as the nuisance caused by the diplopia, surgical evacuation was performed. Current published literatures which are based mostly on case reports describe the various treatment options for subperiosteal haematoma.8-11 Although haematoma can resolve with time, surgical evacuation is the preferred management in most 
cases. ${ }^{8-10}$ In the absence of serious complication, observation of the condition is recommended. ${ }^{9}$ In this instance, careful and close monitoring of optic nerve function and intraocular pressure should be carried out. Blood investigation for coagulopathy is mandatory to look for any bleeding diathesis in these young patients.

Surgical evacuation can be performed with supraorbital incision, ${ }^{9}$ needle aspiration ${ }^{10}$ or even endoscopic surgery. ${ }^{11}$ Apart from being fairly safe and simple method, surgical evacuation enables rapid evacuation of the clot and prevents further complication such as calcification, cyst or secondary infection. The need for surgical evacuation or even needle aspiration may be warranted in cases with either progressive or unresolved proptosis, features of optic nerve compression, rebleeding, associated orbital wall fracture, and disturbing diplopia or in those patients who wish for early recovery. In our case, the decision for surgical management was based on the persistently elevated intraocular pressure as well as unresolved proptosis.

\section{CONCLUSION}

Subperiosteal haematoma should always be suspected when a patient presents with proptosis and diplopia after an episode of maxillofacial trauma. Management should be tailored according to the severity and associated complications. We demonstrate a case of subperiosteal haematoma complicated by high intraocular pressure who responded well to surgical evacuation of the blood clot. The clot evacuation not only preserved the function of the optic nerve, it also hastened recovery and prevented further complication of long standing blood clot.

\section{ACKNOWLEDGEMENT}

The authors thank $\mathrm{Dr}$ Nujaimin Udin, M.S Neurosurgery, a Neurosurgeon in Hospital Sultanah Nur Zahirah, for assissting us in the management of this patient.

\section{REFERENCES}

1. Leovic D, Zubcic V, Kopic M et al. Posttraumatic subperiosteal orbital hematoma.

Craniomaxillofac Surg 2011; 39:131-34.

2. Keiji S, Seiji M, Hiroyuki $O$ et al. Non-traumatic Bilateral Orbital Subperiosteal Hematoma in a Person Who Attempted Suicide by Hanging. Tokai J Exp Clin Med 2014; 39:103-05

3. Kyung IW, Yoon DK. Subperiosteal Hematoma of the Orbit associated with Sinusitis. Korean J. Ophthalmol 1997;11:118-22

4. Landa MS, Landa EH, Levine MR. Subperiosteal hematoma of the Orbit. Ophthal Plast Reconstr Surg 1998; 14:189-92

5. Kenichi A, Reizo T, Yoshishige N.Visual Recovery Following Immediate Decompression of
Traumatic Retrobulbar Hemorrhage via Transcranial Approach. Neurol Med Chir 1998; 38:221-24

6. Mafee MF, Putterman A, Valvasorri GE et al. Orbital Space Occupying Lesion: Role of Computed Tomography and Magnetic Resonance Imaging: An Analysis of 145 Cases. Radiol Clin North Am 1987; 25:529-559

7. Onur K, Zafer O, Suna et al. Intraocular pressure and superior ophthalmic vein blood flow velocity in Graves' orbitopathy: relation with the clinical features. Graefes Arch Clin Exp Ophthalmol 2009; 247:1555-1559

8. Mikami T, Maegawa J, Kuroda MM. Subacute phase treatment of subperiosteal hematoma of the orbit with epidural hematoma in the frontal cranial fossa. BMC Ophthalmol 2012; 28:12-18

9. Yazici B, Gonen T. Posttraumatic subperiosteal hematoma of the orbit in children. Ophthal Plast Reconstruct Surg 2011; 27:33-37

10. Rojas MC, Eliason JA, Frederick DR. Needle Aspiration of a Traumatic Subperiosteal Hematoma of the Orbit. Br J Ophthalmol 2002; 86:593-97

11. Kazuhiro N, Yohei H, Daiki O. Endoscopic Drainage of Orbital Subperiosteal Hematoma Secondary to Acute Rhinosinusitis in a Child. Tohoku J Exp.Med 2014;233, 171-74 